\title{
ONE-SIDED INVARIANT SUBSPACES AND DOMAINS OF UNIQUENESS FOR HYPERBOLIC EQUATIONS ${ }^{1}$
}

\author{
R. W. GOODMAN
}

1. Introduction. Suppose $t \rightarrow U(t)$ is a continuous one-parameter group of unitary operators on a complex Hilbert space $K$, and let $H$ be the self-adjoint generator of this group. Do there exist real or complex linear closed subspaces of $K$ which are invariant under the semigroup $[U(t) \mid t \geqq 0]$ but which are not invariant under the full group? In $\$ 2$ we investigate this question under the additional hypothesis that $H$ be a positive-definite operator. Our basic result, when $H \geqq c I$ $>0$, is that there are no proper one-sided invariant manifolds; the invariant subspaces (real or complex linear) for $[U(t) \mid-\infty<t<\infty]$ are precisely those for $\left[U(t) \mid t_{0}<t<\infty\right], t_{0}$ being an arbitrary real number. This fact is exploited in $\$ 3$ to obtain some sharp results on domains of uniqueness for normalizable (finite-energy) solutions of the Klein-Gordon and related hyperbolic partial differential equations. The general result is that such solutions are uniquely determined by their values on an open time-like backward cone in spacetime. This result carries over to the quantized Klein-Gordon field (see Segal [5]), and it follows that the collection of field operators $R(f)$, with $f$ a testing function supported on an open time-like backward cone, is complete, i.e. bounded functions of these operators are weakly dense in the space of all bounded operators on the field state space.

Lax, Morawetz and Phillips [3] have recently considered scattering for the wave equation, which is the limiting case of the Klein-Gordon equation when the mass $m \rightarrow 0$. An interesting result (cf. our Theorem 3.1) of their investigation is that a finite-energy solution of the wave equation which vanishes in both the forward and backward light cones is zero identically.

The author would like to thank Professor I. E. Segal for many clarifying remarks and Dr. Walter Strauss for pointing out that Theorem 4.2 follows from Theorem 3.1.

Presented to the Society, April 29, 1963, under the title One-sided invariant subspaces and domains of dependence for hyperbolic equations; received by the editors April 15, 1963.

1 This research was supported in part by the National Science Foundation, and is part of the author's doctoral dissertation at the Massachusetts Institute of Technology. 
2. Groups with positive generators. Suppose $t \rightarrow U(t)$ is a oneparameter unitary group on a complex Hilbert space $K$. By Stone's theorem [4] there exists a self-adjoint operator $H$ on $K$ with spectral family $\left[E_{\lambda}\right]$ such that $U(t)=\exp i H t$.

THEOREM 2.1. If $H$ is a strictly positive operator, then any closed real-linear manifold $M$ in $K$ which is invariant under $\left[U(t) \mid t \geqq t_{0}\right]$ is also invariant under $\left[U(t) \mid t<t_{0}\right]$.

Proof. As a preliminary remark, we observe that if $(u, v)$ is the complex inner product on $K$, then $K$ becomes a real Hilbert space $K_{r}$ under the inner product $[u, v]=\operatorname{Re}(u, v)=[(u, v)+(v, u)] / 2$. We shall use the symbol $\perp$ to denote orthogonality in $K_{r}$.

If $u, v$ are in $K$, then by virtue of the positivity of $H$,

$$
(U(t) u, v)=\int_{m}^{\infty} e^{i \lambda t} d\left(E_{\lambda} u, v\right)
$$

with $m$ a fixed positive number. This representation makes it evident that $f(t)=(U(t) u, v)$ can be extended to a holomorphic function in the half-plane $[t+i s \mid s>0]$. Furthermore, $|f(t+i s)| \leqq e^{-m s}\|u\| \cdot\|v\|$.

Suppose now that $u$ belongs to $M$, and $v$ belongs to $M^{\perp}$. By hypothesis $U(t) u$ also belongs to $M$, if $t \geqq t_{0}$, hence $\operatorname{Re} f(t)=0, t \geqq t_{0}$. To complete the proof of Theorem 2.1 it suffices to show that such an $f$ must be identically zero. For, if $f \equiv 0$, then $v \perp u$ implies $v \perp U(t) u$ for all $t . M$ is a closed subspace of $K_{r}$, so that for any $t$,

$$
U(t) M \subset\left(M^{\perp}\right)^{\perp}=M .
$$

It remains to prove the

Lemma. Let $f$ be analytic for $\operatorname{Im} z>0$, continuous for $\operatorname{Im} z \geqq 0$. Suppose further that $|f(x+i y)| \leqq C e^{-m y}$ for some $m>0$ and all $x$. Then $\operatorname{Re} f(x)=0$ for all $x>t_{0}$ implies that $f=0$.

Proof. Under the hypotheses we may apply the Schwartz reflection principle to continue $f$ analytically as a bounded function in $\operatorname{Re} z>t_{0}$ by defining $f(x-i y)=-f(x+i y)^{*}$. It follows that $f\left(t_{0}+i y\right)$ $=O\left(e^{-m|y|}\right)$. By a Phragmen-Lindelöf theorem $[8, \S 5.8], f(z)=0$ identically in the half-plane, $\operatorname{Re} z \geqq t_{0}$, hence also in the upper half-plane.

3. Klein-Gordon equation and domains of uniqueness. An important physical system in relativistic quantum mechanics is the quantized scalar meson field [6], whose state space of inputs and outputs (the asymptotic free field) is built up from normalizable solutions of the Klein-Gordon equation 


$$
\square \phi=m^{2} \phi,
$$

where $m>0$ and $\square$ is the wave operator $\Delta-\partial^{2} / \partial t^{2}$. The Hilbert space $K_{m}$ of real normalizable solutions of this equation may be described most succinctly via Fourier analysis as follows (see [5]): Identifying $x_{0}$ with $t$, we let $x=\left(x_{0}, x_{1}, \cdots, x_{n}\right)$ denote a vector in $R^{n+1}$ and $k=\left(k_{0}, k_{1}, \cdots, k_{n}\right)$ a vector in the dual space, with $k \cdot x$ $=k_{0} x_{0}-k_{1} x_{1}-\cdots-k_{n} x_{n}$. Consider the complex-valued functions on the hyperboloid $k \cdot k=m^{2}$ which are measurable and square-summable with respect to the Lorentz-invariant measure $d \chi(k)=\left|k_{0}\right|^{-1} d_{n} k$, $d_{n} k$ denoting $n$-dimensional Lebesgue measure $d k_{1} d k_{2} \cdots d k_{n}$. To such a function $f$ corresponds a (generalized) solution $\phi$ given by

$$
\phi(x)=\int e^{i k \cdot x} f(k) d \chi(k) .
$$

So that $\phi$ be real-valued, we require that $f(-k)=f(k)^{*} ; K_{m}$ is then the real Hilbert space of all such Hermitian-symmetric, square-summable $f$, with inner product

$$
[f, g]=\int f(k) g(-k) d \chi(k)
$$

which is always real.

The invariance of the wave operator $\square$ under the inhomogeneous Lorentz group yields an orthogonal representation of this group on $K_{m}$; in particular, time translations, $x \rightarrow x+t e_{0}, e_{0}$ a unit vector on the $x_{0}$-axis, give rise to a one-parameter orthogonal group

$$
U(t): f(k) \rightarrow e^{i k_{0} t} f(k), \quad f \in K_{m} .
$$

A distinguished property of this representation, for $m>0$, is that a complex structure may be put on $K_{m}$ such that the representation of the Lorentz group becomes unitary, and $U(t)=e^{i t H}$ with selfadjoint generator $H \geqq m$. (See Segal [6].)

Explicitly, let $j$ be the orthogonal transformation on $K_{m}$ sending $f(k) \rightarrow i \operatorname{sgn}\left(k_{0}\right) f(k)$; then $j^{2}=-I$, and $j$ commutes with $U(t)$. By defining a complex inner product

$$
(f, g)=[f, g]-i[j f, g]
$$

and multiplication by complex scalars

$$
(\alpha+i \beta) f=\alpha f+\beta j f,
$$

we make $K_{m}$ into a complex Hilbert space. The operator $H$ is given 
by multiplication by $\left|k_{0}\right|$, and since $k \cdot k=m^{2}$, it follows that the spectrum of $H=$ range $\left|k_{0}\right|=[m, \infty)$.

TheOREM 3.1. If $\phi$ is a real-valued normalizable solution of $\square \phi$ $=m^{2} \phi$, vanishing on an open time-like cone, then $\phi=0$ identically.

Proof. By a time-like cone is meant a cone in $R^{n+1}$ containing a time-like vector $x\left(x_{0}^{2}>x_{1}^{2}+\cdots+x_{n}^{2}\right)$. Since $K_{m}$ is invariant under a change of coordinates in $R^{n+1}$ given by a Lorentz transformation, it is enough to consider the case $\phi=0$ on an open cone $C$ containing the negative $x_{0}$-axis. If $\phi$ corresponds to $f$ via (3.1), then the hypothesis is equivalent to $[f, P \Psi]=0$ for every real-valued function $\Psi \in C_{0}^{\infty}\left(R^{n+1}\right)$ with compact support in $C . P \Psi$ here denotes the projection of $\Psi$ onto $K_{m}$, i.e. the restriction of the Fourier transform of $\Psi$ (in $R^{n+1}$ ) to the hyperboloid $k^{2}=m^{2}$.

Let now $M=\left[P \Psi \mid \Psi \in C_{0}^{\infty}\left(R^{n+1}\right)\right.$, Supp $\left.(\Psi) \subset C\right]-$, the bar signifying closure in $K_{m}$. By continuity $[f, g]=0$ for all $g \in M$; furthermore, $M$ is invariant under $[U(t) \mid t \geqq 0]$, since $U(t) P \Psi=P \Psi_{t}$, with $\Psi_{t}(x)$ $=\Psi\left(x+t e_{0}\right)$. Thus $M$ is a one-sided invariant manifold and by Theorem 2.1 we conclude that $U(t) M \subset M$ for all $t$. For any $\Psi \in C_{0}^{\infty}\left(R^{n+1}\right)$, however, there exists a $t>0$ such that Supp $\left(\Psi_{t}\right) \subset C$. Hence $P \Psi$ $=U(-t) P \Psi_{t} \in M$, and so $[f, P \Psi]=0$ for all test functions $\Psi$, implying that $f=0$.

It follows from the proof that we have

Corollary 3.1. If $M$ is the set of all $C^{\infty}$ functions on space-time with compact support contained in a fixed open time-like cone, then the projection of $M$ onto $K_{m}$ is dense in $K_{m}$.

Remarks. Theorem 3.1 is a sharp result in two directions. In the $m=0$ case, where the energy operator is not strictly positive, there exist nonzero normalizable solutions to the wave equation,

$$
\square \phi=0 \text {, }
$$

which vanish in the backward light cone. Furthermore, nonzero $C^{\infty}$ solutions of the Klein-Gordon equation exist which vanish in the backward cone by virtue of familiar general principles concerning hyperbolic equations. (See Courant-Hilbert [1, pp. 450-459] for a discussion of this characteristic Cauchy problem.) Thus we see that the physically-motivated requirements of normalizability and positivity of the energy force a solution to be determined everywhere by its values on any open time-like cone.

4. Klein-Gordon equation with perturbations. Our preceding result on domains of uniqueness for normalizable solutions of the KG 
(Klein-Gordon) equation is also valid for a class of linear time-independent perturbations, and nonlinear time-dependent perturbations of the equation. In the first case, where the perturbation consists of a non-negative potential $V(x)$, an abstraction of the proof used for the $\mathrm{KG}$ equation establishes the result. In the second case, where the perturbation is, e.g., a continuous time-dependent operator small at $t= \pm \infty$, results of Walter Strauss [7] on nonlinear scattering allow the result for the KG equation to be used, but with the weaker conclusion that only the full backward light cone is a domain of uniqueness for normalizable solutions of the nonlinear equation.

Let us consider first the KG equation with potential, viz.,

$$
\square \phi=\left(m^{2}+V\right) \phi,
$$

where $V$ is an a.e. non-negative measurable function of the space variable $x$, and $m^{2}>0$. To avoid irrelevant complications, we shall assume that any singularities of $V$ are mild enough so that the operator $A_{0}=-\Delta+m^{2}+V$, with $D\left(A_{0}\right)=\mathcal{S}$ (the Schwartz space of rapidly decreasing functions) is essentially self-adjoint on $K$, the real Hilbert space of real-valued Lebesgue square-summable functions on $R^{n}$ (see Kato [2]). We denote the closure of $A_{0}$ by $A ; A$ is then self-adjoint and is easily seen to satisfy $A \geqq m^{2} I$. By the spectral theorem $B=A^{1 / 2}$ exists as a positive self-adjoint operator.

To obtain a Hilbert space structure on solutions of (4.1), we introduce the real Hilbert space $\mathcal{F}_{0}=D(A) \oplus D(B)$, with the inner product

$$
(u, v)_{\mathfrak{H C}_{0}}=\left(A u_{1}, A v_{1}\right)_{K}+\left(B u_{2}, B v_{2}\right)_{K}
$$

when

$$
u=\left(\begin{array}{l}
u_{1} \\
u_{2}
\end{array}\right) \text { and } v=\left(\begin{array}{l}
v_{1} \\
v_{2}
\end{array}\right)
$$

Writing (4.1) in the abstract form

$$
d^{2} \phi / d t^{2}=-A \phi,
$$

we define the space $\mathcal{H C}$ of normalizable solutions of (4.2) as the set of all $K$-valued functions of $t$ satisfying (i) $t \rightarrow \phi(t)$ is strongly differentiable and the derivative $\phi^{\prime}(t)$ is absolutely continuous and a.e. strongly differentiable, (ii) $\phi(0) \in D(A)$ and $\phi^{\prime}(0) \in D(B)$, (iii) $\phi$ satisfies (4.2) a.e.

LEMMA 4.1. Every element of $\mathfrak{H C}$ has the unique representation 


$$
\left(\begin{array}{l}
\phi(t) \\
\phi^{\prime}(t)
\end{array}\right)=\left(\begin{array}{cc}
\cos t B & B^{-1} \sin t B \\
-B \sin t B & \cos t B
\end{array}\right)\left(\begin{array}{l}
\phi(0) \\
\phi^{\prime}(0)
\end{array}\right) .
$$

(See Strauss [7].)

From Lemma 4.1 we see that each element $\phi$ of $\mathfrak{H C}$ with Cauchy data $\phi(0)=\phi_{0}, \phi^{\prime}(0)=\phi_{1}$ at time $t=0$ corresponds uniquely to the element

$$
\left(\begin{array}{l}
\phi_{0} \\
\phi_{1}
\end{array}\right)
$$

of $\mathfrak{H}_{0}$. If the matrix operator in (4.3) is denoted by $U_{t}$, then $t \rightarrow U_{t}$ is a continuous one-parameter orthogonal group on $\mathcal{H C}_{0}$ that maps the Cauchy data of $\phi$ at $t=0$ into the Cauchy data of $\phi$ at time $t$.

THEOREM 4.1. If $\phi$ is a normalizable solution of (4.2) and $\mathfrak{e}$ is an open cone in $R^{n+1}$ with vertex on the t-axis and containing a semiinfinite segment of the $t$-axis, then $\phi=0$ on $\mathcal{C}$ implies $\phi \equiv 0$.

Proof. By hypothesis

$$
\langle\phi, u\rangle \equiv \int_{R^{n+1}} \phi(x, t) u(x, t) d x d t=0
$$

for all $u \in C_{c}^{\infty}(\mathcal{e})$, and the conclusion of the theorem follows if we show that (4.4) must then hold for all $u \in C_{c}^{\infty}\left(R^{n+1}\right)$. For this purpose we need the following construction:

Let $J$ be the operator on $\mathcal{H C}$ which acts formally as the Hilbert transform with respect to time, i.e.

$$
J\left(\cos t B \phi_{0}+B^{-1} \sin t B \phi_{1}\right)=-\sin t B \phi_{0}+B^{-1} \cos t B \phi_{1} .
$$

Equivalently, in terms of its action on the space $\mathcal{F}_{0}$ of Cauchy data at $t=0, J$ corresponds to the matrix operator

$$
j=\left(\begin{array}{rr}
0 & B^{-1} \\
-B & 0
\end{array}\right) \text {. }
$$

Lемма 4.2. (a) $j$ is an isometric transformation on $\mathfrak{H}_{0}$ satisfying $j^{*}=-j, j^{2}=-I$.

(b) $j U_{t}=U_{t} j$.

Proof. Direct calculation.

By Lemma 4.2 , just as in the case of the $\mathrm{KG}$ equation in $\$ 3$, we can define a complex structure on $\mathcal{F}_{0}$ via $j$, and $t \rightarrow U_{t}$ becomes a oneparameter unitary group on the complex Hilbert space $\mathcal{F}_{c}$. The selfadjoint generator $H$ of $U_{t}$ is obtained as 


$$
\lim _{t \rightarrow 0}(j t)^{-1}\left(U_{t}-I\right)=\left(\begin{array}{ll}
B & 0 \\
0 & B
\end{array}\right),
$$

with $D(H)=D\left(B^{3}\right) \oplus D\left(B^{2}\right)$. Since $H$ commutes with $j$, and $B \geqq m I$ on $K$, it follows that $H \geqq m I$ on $\mathcal{H}_{c}$.

We now return to the proof of Theorem 4.1. From Theorem 2.1 and the construction above, it follows that the real-linear span in $\mathfrak{H C}_{0}$ of the Cauchy data of $\phi$ at times $t$ belonging to any semi-infinite interval coincides with the span of the Cauchy data of $\phi$ at all times $-\infty<t<+\infty$. Since $\phi \rightarrow\langle\phi, u\rangle$ (defined in Equation (4.4)) is a continuous linear functional on $\mathcal{F}_{0}$, we conclude, as in the proof of Theorem 3.1 , that $\langle\phi, u\rangle=0$ for all $u \in C_{0}^{\infty}\left(\mathfrak{e}_{t}\right),-\infty<t<\infty$, where $\mathfrak{e}_{t}$ is the translated cone $t e_{0}+\mathcal{C}$. Any compact set in $R^{n+1}$ is contained in some $\mathfrak{C}_{t}$, however, so $\phi \equiv 0$.

Nonlinear perturbations. The case of the equation

$$
\left(\square-m^{2}\right) u=L(u)
$$

with $L$ a possibly nonlinear and time-dependent operator can be successfully treated whenever scattering theory exists for (4.5), considered as a perturbation of the free $\mathrm{KG}$ equation

$$
\left(\square-m^{2}\right) u=0 \text {. }
$$

(Strauss [7] has discussed the nonlinear scattering problem, and has obtained some sufficient conditions on $L$ for existence of the wave operators.) Recall that the wave operators $W_{ \pm}$are constructed as follows: If $u(t)$ is a normalizable solution of (4.5), consider the function $u_{s}$, obtained as the solution to the KG equation with Cauchy data $u(s), u^{\prime}(s)$ at time $t=s$. Then $W_{ \pm} u=\lim _{s \rightarrow \pm \infty} u_{s}$, assuming that this strong limit in the Hilbert space of normalizable KG solutions exists.

Theorem 4.2. If $W_{-}$exists and is $1-1$, and $u$ is a normalizable solution of (4.5) vanishing on the solid backward light cone, then $u=0$.

If $W_{+}$exists and is $1-1$, and $u$ is a normalizable solution of (4.5) vanishing on the solid forward light cone, then $u=0$.

This theorem is an immediate consequence of our earlier results and the following

LemмA. If $u=0$ on the backward (forward) light cone, then so does $W_{-} u\left(W_{+} u\right)$.

Proof. Let $\mathfrak{C}$ denote the solid backward light cone, $\mathfrak{C}_{s}$ the cone translated through time $s$, and $D_{s}=\mathfrak{e} \cap-\mathfrak{C}_{8}$. With $u_{s}$ defined as above, the vanishing of $u$ on $\mathfrak{C}$ and the hyperbolic propagation prop- 
erty of the KG equation imply that $u_{s}=0$ on $D_{s}$ for all $s<0$. Hence $W_{-} u$ vanishes on $\mathrm{e}=U_{s<0} D_{s}$.

(The same proof works for $W_{+} u$, of course.)

5. Causal algebras of field operators. Consider the quantized free scalar meson field of mass $m>0$. (See [5].) Mathematically, we have a map $f \rightarrow R(f)$ from $C_{c}^{\infty}\left(R^{4}\right)$ to self-adjoint operators on a complex Hilbert space $K$ satisfying the usual physical desiderata (commutation rules, Lorentz-transformation properties, irreducibility) and certain continuity requirements. Let $W(f)=\exp [i R(f)]$, and denote by Pf the projection of $f \in C_{c}^{\infty}\left(R^{4}\right)$ onto the KG Hilbert space, i.e. the restriction of the Fourier transform of $f$ to the mass hyperboloid $k^{2}=m^{2}$. By hypothesis, the set of operators $[W(f)]$ is irreducible on $K$, and the map $f \rightarrow W(f)$ is continuous with respect to the Lorentzinvariant Hilbert topology on $P f$ and the weak operator topology on $W(f)$.

THEOREM 5.1. If $R(\cdot)$ is the quantized field for the KG equation of mass $m>0$, then the operators $R(g)$, with Supp $(g)$ contained in a fixed open time-like cone, generate all bounded operators on the field state space $K$.

Proof. From Corollary 3.1, the set of all such $g$ is strongly dense in the KG Hilbert space $K_{m}$. Hence by the continuity of the map $f \rightarrow W(f)$, it follows that the ring of operators generated by $W(g)$, $g$ ranging over a dense subset of $K_{m}$, is $B(K)$.

\section{BIBLIOGRAPHY}

1. R. Courant and D. Hilbert, Methods of mathematical physics. II, 2nd ed., Interscience, New York, 1962.

2. T. Kato, Fundamental properties of Hamiltonian operators of Schrödinger type, Trans. Amer. Math. Soc. 70 (1951), 195-211.

3. P. D. Lax, C. S. Morawetz and R. S. Phillips, The exponential decay of solutions of the wave equations in the exterior of a star-shaped obstacle, Bull. Amer. Math. Soc. 68 (1962), 593-595.

4. L. H. Loomis, Abstract harmonic analysis, Van Nostrand, New York, 1953.

5. I. E. Segal, Foundations of the theory of dynamical systems of infinitely many degrees of freedom. II, Canad. J. Math. 13 (1961), 1-18.

6. - Mathematical problems of fundamental relativistic physics, Lectures in Applied Mathematics 2, Amer. Math. Soc., Providence, R. I., 1962.

7. W. Strauss, Scattering for hyperbolic equations, Trans. Amer. Math. Soc. 108 (1963), 13-37.

8. E. C. Titchmarsh, The theory of functions, 2nd ed., Oxford Univ. Press, London, 1939.

Massachusetts Institute of Technology 